\title{
Secure Serverless Computing using Dynamic Information Flow Control
}

KALEV ALPERNAS, Tel Aviv University, Israel and VMware Research, USA

CORMAC FLANAGAN, UC Santa Cruz, USA

SADJAD FOULADI, Stanford University, USA

LEONID RYZHYK, VMware Research, USA

MOOLY SAGIV*, Tel Aviv University, Israel

THOMAS SCHMITZ, UC Santa Cruz, USA

KEITH WINSTEIN, Stanford University, USA

The rise of serverless computing provides an opportunity to rethink cloud security. We present an approach for securing serverless systems using a novel form of dynamic information flow control (IFC).

We show that in serverless applications, the termination channel found in most existing IFC systems can be arbitrarily amplified via multiple concurrent requests, necessitating a stronger termination-sensitive non-interference guarantee, which we achieve using a combination of static labeling of serverless processes and dynamic faceted labeling of persistent data.

We describe our implementation of this approach on top of JavaScript for AWS Lambda and OpenWhisk serverless platforms, and present three realistic case studies showing that it can enforce important IFC security properties with modest overhead.

CCS Concepts: • Security and privacy $\rightarrow$ Information flow control; Access control;

Additional Key Words and Phrases: Information Flow Control, Serverless, Cloud Computing

\section{ACM Reference Format:}

Kalev Alpernas, Cormac Flanagan, Sadjad Fouladi, Leonid Ryzhyk, Mooly Sagiv, Thomas Schmitz, and Keith Winstein. 2018. Secure Serverless Computing using Dynamic Information Flow Control. Proc. ACM Program. Lang. 2, OOPSLA, Article 118 (November 2018), 26 pages. https://doi.org/10.1145/3276488

\section{INTRODUCTION}

In May 2017, the Equifax credit reporting agency suffered a security breach, leaking social security numbers and other personal information of 145.5 million consumers [Forbes 2017]. The breach, which exploited a code injection vulnerability in Apache Struts [National Vulnerability Database 2017], became the latest in a series of high-profile attacks on public and private clouds compromising sensitive personal information of hundreds of million users [Computerworld 2009, 2014; Digital Trends 2016; Forbes 2014; The Register 2011; Wikipedia 2017a,b,c; Wired 2016; ZDNet 2015; ZDNet 2016].

${ }^{*}$ Work done while at VMware Research.

Authors' addresses: Kalev Alpernas, Tel Aviv University, Israel, VMware Research, USA, kalevalp@post.tau.ac.il; Cormac Flanagan, UC Santa Cruz, USA; Sadjad Fouladi, Stanford University, USA; Leonid Ryzhyk, VMware Research, USA; Mooly Sagiv, Tel Aviv University, Israel; Thomas Schmitz, UC Santa Cruz, USA; Keith Winstein, Stanford University, USA.

This work is licensed under a Creative Commons Attribution 4.0 International License.

(C) 2018 Copyright held by the owner/author(s).

2475-1421/2018/11-ART118

https://doi.org/10.1145/3276488

Proc. ACM Program. Lang., Vol. 2, No. OOPSLA, Article 118. Publication date: November 2018. 
Most of these attacks can be traced down to two types of faults: misconfigurations and software vulnerabilities. The former include issues like incorrect database security attributes [Digital Trends 2016; PCWorld 2010; TechRepublic 2017], the choice of weak authentication schemes [Computerworld 2014], or the use of unpatched software [Forbes 2017]. The latter include code and SQL injections, file inclusions, directory traversals, etc. [CNET Magazine 2011; Computerworld 2009; ZDNet 2015; ZDNet 2016].

Simply put, the enormous Trusted Computing Base (TCB) of modern cloud applications makes it intractable to enforce information security in these environments.

A promising avenue to a smaller TCB lies in the use of information flow control (IFC)-based security [Denning 1976; Sabelfeld and Myers 2003]. In the IFC world, information is protected by a global security policy that cannot be overridden by a misconfigured application. The policy explicitly and concisely captures constraints on end-to-end information flow through the system, e.g., "credit card numbers can only be exposed to appropriate card associations (e.g., Visa or MasterCard)".

The IFC system enforces the policy even for buggy or malicious applications, thus removing application code and configuration from the TCB of the cloud. In particular, an application that has been hijacked by a code injection attack should not be able to bypass the enforcement mechanism. This is in contrast to security models based on access control lists or capabilities, where, for instance, a compromised program running with database administrator privileges can easily leak the entire database to a remote attacker.

Despite significant progress on IFC, it remains difficult to apply in real software. Dynamic IFC systems [Austin and Flanagan 2009; De Groef et al. 2012; Efstathopoulos et al. 2005; Stefan et al. 2011] often incur high runtime overhead. Static IFC (see e.g. [Myers 1999; Myers and Liskov 2000; Sabelfeld and Myers 2003; Zdancewic 2002]) systems shift the costs to development time, usually via the use of type systems; however, they restrict the style of programming, which complicates their adoption.

We demonstrate that IFC for cloud computing is feasible, and can be implemented for essentially unmodified applications with manageable overhead. We achieve these properties by leveraging recent developments in cloud computing, namely, the rise of serverless computing [Sbarski 2017]. Initially popularized by Amazon's AWS Lambda [Amazon 2017a], serverless computing is rapidly gaining adoption by cloud providers [Apache Software Foundation 2017; Fn Project 2017; Google 2017; IBM 2017; Microsoft 2017] and tenants [Airbnb 2017; Baird et al. 2016; Boyd 2017; Chowhan 2016; Ellis 2017; Eriksen 2013; Fouladi et al. 2017b; Jonas et al. 2017; Nordstrom Technology 2017] due to its key benefits: elastic scalability, ease of deployment, and flexible pay-per-use pricing.

Serverless computing achieves these benefits by decoupling application logic from resource management. In the serverless model, users express their applications as collections of functions triggered in response to user requests or calls by other functions. A function can be written in any language and may request a certain runtime environment, including, e.g., specific versions of the Python interpreter and libraries. However, the function is agnostic of where this environment is instantiated: a physical machine, a virtual machine or a container. The cloud platform manages function placement and scheduling, automatically spawning new function instances on demand. This requires application state to be decoupled from functions and placed in a shared data store (e.g., a database or a key-value store), allowing all function instances to access the state regardless of their physical placement in the cloud.

We argue that serverless computing has fundamental implications for cloud security. In particular, it may enable practical IFC for the cloud. Our key observation is that a serverless function constitutes a natural unit of information flow tracking. First, a serverless function activation handles a single request on behalf of a specific user and only accesses secrets related to this request. Second, each invocation starts from a clean state and does not get contaminated with sensitive data from previous

Proc. ACM Program. Lang., Vol. 2, No. OOPSLA, Article 118. Publication date: November 2018. 


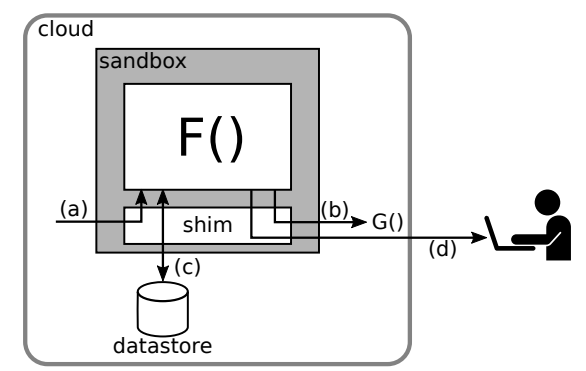

Fig. 1. Trapeze architecture. Serverless function $F$ is encapsulated in a sandbox. All inputs and outputs of $F$, including (a) invocations of $F$ from within and from outside of the cloud, (b) calls to other serverless functions, (c) reads and writes to shared data stores, and (d) external communication channels, are monitored by the security shim.

invocations; any state shared across invocations must be kept in a global data store ${ }^{1}$. Under a conservative assumption that all secrets obtained during function execution propagate to all its outputs, we can track the global flow of information in the system by monitoring inputs and outputs of all functions in the system.

Based on this observation, we develop the first IFC system for serverless applications, called Trapeze. Trapeze encapsulates each unmodified serverless function in a sandbox, which intercepts all interactions between the function and the rest of the world, including other functions, shared data stores, and external communication channels, and redirects them to the security shim (Figure 1). The shim tracks information flow and enforces the global security policy.

The class of supported policies, along with policy enforcement rules, is defined by Trapeze's dynamic IFC model. The model addresses a weakness in many existing static and dynamic IFC systems, which leak information through the termination channel, whereby an adversary can infer one bit of information by observing termination or non-termination of the program. The parallel nature of the serverless environment amplifies this weakness, allowing the attacker to construct a high-bandwidth information channel, effectively defeating the purpose of IFC (Section 2).

Our IFC model eliminates this channel by enforcing a strong security property known as termination-sensitive non-interference (TSNI) [Sabelfeld and Sands 2001]. Trapeze achieves TSNI through a novel combination of static program labeling with dynamic labeling of the data store based on a faceted store semantics. Static program labeling restricts the sensitivity of data a serverless function can observe ahead of time and is key to eliminating the termination channel. Dynamic data labeling is crucial to securing unmodified applications that do not statically partition the data store into security compartments, while the faceted store semantics eliminates implicit storage channels. We present a formal proof, validated using the Coq proof assistant, that our model enforces TSNI.

We evaluate Trapeze on three real-world serverless applications: an online retail store [Nordstrom Technology 2017], a parallel build system [Fouladi et al. 2017a], and an image feature extraction service [Serverless, Inc 2017]. We use Trapeze to secure these applications with minimal changes to application code.

Thus, our key contributions in this work are

(1) a light-weight IFC shim architecture for serverless computing,

\footnotetext{
${ }^{1}$ Practical serverless platforms do not strictly enforce this property, allowing functions to cache state across invocations. In Section 6 we discuss how we enforce IFC in the presence of such local cached state.
} 


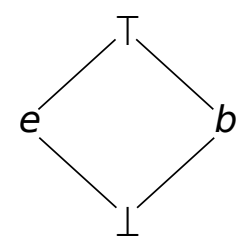

(a) Example security lattice. $T$ is the most secret label at the top of the lattice; $\perp$ is the bottom (least secret) label.

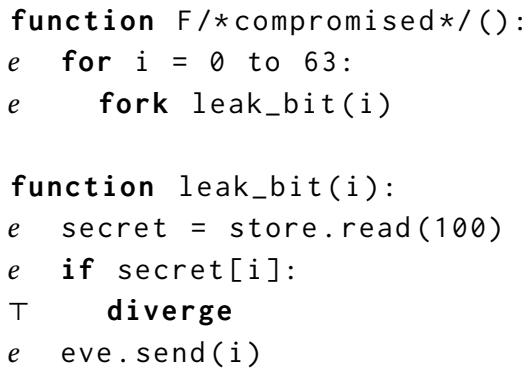

(b) High-bandwidth termination channel in the classical dynamic IFC model.

Fig. 2. Security lattice and program used to demonstrate a high bandwidth termination channel.

(2) a new IFC model that enforces TSNI, along with its formal semantics and proof of correctness, and

(3) an experimental evaluation of the architecture and the model on three serverless applications.

Finally, we point out that our IFC model is not limited to the serverless domain. Generally speaking, it applies to any reactive system that decouples computation from state. Examples of such systems include, e.g., Hadoop [Apache Software Foundation [n. d.]], Apache Spark [Zaharia et al. 2012] and Stateless Network Functions [Kablan et al. 2017].

\section{WHY A NEW IFC MODEL FOR SERVERLESS?}

As discussed above, a serverless function offers a convenient unit of information tracking, enabling practical IFC for serverless applications. It seems natural that our next step should be adapting an existing IFC model to the serverless environment. However, we do not take this path, as existing models do not provide adequate security for serverless applications. Specifically, most previous IFC models, both dynamic and static, enforce a security property known as termination-insensitive non-interference (TINI) [Sabelfeld and Sands 2001]. Intuitively, TINI guarantees that an attacker cannot deduce secrets stored in the system from its non-secret outputs. However, they may be able to deduce part of a secret from the fact that the system stopped producing outputs.

This information channel, known as the termination channel, is often disregarded because it has low bandwidth, typically leaking just a single bit. This is not true in serverless systems. Below, we construct an attack on a serverless application, that amplifies the termination channel by spawning many parallel computations, each leaking one bit.

Example 2.1. Consider a serverless system with two users: a benign user Bob and a malicious user Eve. We introduce security labels $b$ and $e$ to tag Bob's and Eve's data respectively. Labels form a lattice, with labels higher up in the lattice representing more secret data. Bob and Eve are mutually distrusting, therefore their labels are incomparable (Figure 2a).

Eve launches a code injection attack against serverless function $F$, forcing the function to execute malicious code in Figure 2b. This code is designed to leak Bob's 64-bit secret stored under key 100 in the key-value store to Eve.

We assume that the system is secured using a classical dynamic IFC model where the function's label gets bumped up when branching or looping on a secret and gets bumped back down when the control flow structure ends. The function initially runs with Eve's label $e$ (every line in the listing is annotated with the current label of the function). Before reading Bob's secret, the function forks 64 instances of the helper function leak_bit (lines 2-3). Each instance reads the secret and 
branches on one of its bits. If the bit is 1 , then the function's dynamic label rises to $b \sqcup e=\top$ and the function goes into an infinite loop. Otherwise, if the bit is 0 , then the function's label remains at $e$ and the function sends a message to Eve (here, eve is a handle to Eve's HTTP session), which succeeds because the label of the channel $(e)$ is higher than or equal to the function's current label Eventually, Eve receives a list of the bits of the secret that are equal to 0 .

We implemented the above attack in AWS Lambda and were able to leak 170 bits per second by scaling the number of threads. Thus, by elastically scaling the computation, the serverless architecture also scales the termination channel. This is in line with theoretical results, which suggest that in a concurrent system, the termination channel can leak a secret in time linear in the size of the secret [Askarov et al. 2008].

We therefore aim for a stronger security guarantee, known as termination-sensitive noninterference (TSNI) [Sabelfeld and Sands 2001], which eliminates the termination channel. Note that the termination channel in the above example arises as the function's label, and hence its ability to send to an external channel, depends on the labels of values the function reads from the store. In contrast, our proposed model assigns a static security label to each function activation. To do so, we take advantage of the fact that a serverless function always runs on behalf of a specific user and can be assigned a corresponding security label. The complete model, presented in Sections 3 and 4, also offers a secure way to dynamically increase the function's label without introducing a side channel.

The function's label determines its view of the data store: the function can only observe the existence of data whose label does not exceed the function's label. For example, when reading a key that contains a secret above function's current label, the store returns the same result as if the key was not present in the store. This information hiding semantics is somewhat tricky to maintain when multiple functions with incomparable labels write to the same store location. We avoid information leaks in this situation by employing faceted store semantics, where each record can contain several values (facets) with different security labels [Austin and Flanagan 2012; Yang et al. 2016].

To the best of our knowledge, Trapeze is the first IFC system to combine static program labeling with dynamic labeling of data using faceting. This combination eliminates termination and storage channels and enforces a strong security property, TSNI.

\section{INFORMAL DESIGN}

\subsection{Threat Model and Assumptions}

We assume that the following entities are trusted, i.e., not malicious or compromised: (1) the cloud operator, (2) physical hosts and network, (3) system software (OS, hypervisor, container manager, scheduler), (4) serverless runtime, (5) shared data stores, (6) the sandboxing technology. Assumptions (1) through (5) can in the future be relaxed with the help of a secure enclave technology such as Intel SGX [Hunt et al. 2016; Intel Corporation 2014], data encryption, and software verification [Denning and Denning 1977; Heintze and Riecke 1998].

We further trust the serverless application administrator to enforce the following invariants on application configuration: (1) all data stores used by the application are configured to only be accessible from serverless functions, (2) all serverless functions in the system are sandboxed.

Finally, we trust the application developer to correctly define the application's information flow policy and declassifier functions (Section 6.2).

The rest of the application is untrusted. In particular, we assume that the attacker can compromise application code running inside serverless functions, including any of the frameworks and libraries it uses. 
In this paper we focus on data confidentiality, i.e., protecting sensitive data from being exposed to unauthorized users. The complementary problem of enforcing data integrity, i.e., protecting data from unauthorized modification is outside the scope of Trapeze, although it can also be enforced with the help of IFC techniques [Sabelfeld and Myers 2003].

\subsection{Security Lattice}

We start the construction of our IFC model with the lattice of security labels. Labels represent security classes of information flowing through the system. Trapeze does not assign any specific semantics to labels; however in practice they typically represent users or roles of the system.

Trapeze relies on a trusted authentication gateway to tag all external input and output channels with correct security labels. For example, when Eve establishes an HTTP session with the system, the session gets tagged with Eve's label $e$.

Given the labeling of inputs and outputs, Trapeze applies information flow rules presented below to enforce that information received through an input channel labeled $l_{i}$ can only be exposed through an output channel labeled $l_{o}$ if $l_{i} \sqsubseteq l_{o}$.

\subsection{Information Flow Rules}

The choice of information flow rules determines two critical properties of an IFC system: security and transparency. The former characterizes the class of insecure behaviors the system prevents. The latter characterizes the class of secure programs that the system executes with unmodified semantics and that therefore do not need to be modified to work with Trapeze. Trapeze enforces the strong security property of TSNI at the cost of some loss of transparency that, we argue, is acceptable in serverless systems.

Trapeze assigns a runtime security label to every serverless function activation. This label is derived from the event that triggered the function. In particular, if the function was invoked via an HTTP request from a user, it obtains the user's security label. Alternatively, when invoked by another function, it inherits the caller's label. The function's label controls its ability to send to an output channel: a send is only allowed if the function's label is smaller than or equal to the channel label.

Trapeze also dynamically labels records in the data store. To this end, the security shim intercepts data store operations issued by the function and modifies them to insert and check security labels. When a function creates or updates a record in the store, the record inherits the function's label (see detailed write semantics below). When reading from the store, the function only observes values whose labels are below or equal to its own label. From the function's perspective, the store behaves as if it did not contain any data that the function may not observe.

A function can upgrade its label to an arbitrary higher label using the raiseLabel operation. This operation does not introduce an unauthorized information channel, as the decision to upgrade cannot depend on secrets above function's previous label (such secrets are simply invisible to the function). The upgrade mechanism is useful, for example, when a function running on behalf of a regular user needs to update global statistics on behalf of a superuser. Upgrade is a one-way operation: a function's label can never be downgraded below its current value.

Store semantics Trapeze's security shim conceals the existence of data whose security label is not less than or equal to the function's label. Maintaining this semantics is straightforward when all writes to a data store location carry the same label. Writes with conflicting labels, on the other hand, may introduce implicit storage channels, where the attacker infers secrets by observing that labeled values exist within particular store locations without observing the actual values [Austin and Flanagan 2010]. The following example illustrates the problem: 

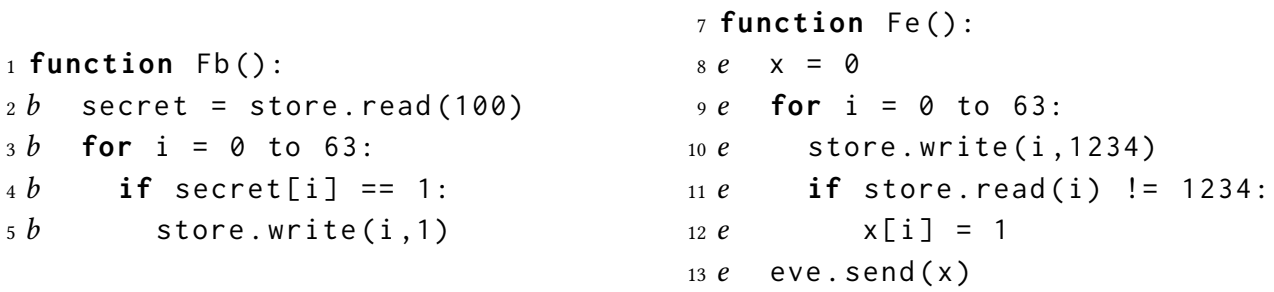

Fig. 3. Implicit storage channel via conflicting writes.

Example 3.1 (Implicit storage channel). Figure 3 shows two functions running with labels $b$ and $e$ respectively that collude to leak Bob's 64-bit secret to Eve. $F b$ reads the secret in line 2; however it does not have the authority to send it to Eve directly. Instead it encodes each bit of the secret using a record in the key-value store. $F e$ reconstructs the secret by attempting to write to locations 0 through 63 and then reading the value back in. There are several ways an IFC system can handle the write-read sequence in lines 10-11, but none of them prevents the unauthorized information flow. The system may fail or diverge on writing to a store location with an incomparable label, thus introducing a termination channel, which can be further amplified by concurrency. Alternatively, it may silently ignore the conflicting write, in which case the subsequent read reveals that the store does not contain the expected value and thus gives away the presence of a secret value at this location. Note that dynamically raising the label of $F e$ to $e \sqcup b$ in line 11 does not prevent the information leak: the failure to send to Eve in line 13 would leak one bit to her. This is another form of the termination channel and can be easily amplified by exploiting concurrency. Finally, we could overwrite Bob's data in the store, but this would introduce an unauthorized channel in the reverse direction, from Eve to Bob.

Note that in the special case when security labels are totally ordered implicit storage channels can be eliminated by applying the no-sensitive-upgrade rule [Austin and Flanagan 2010], which allows writes with a low label to a high location, but fails writes with high labels to low locations However, no such mechanism exists for writes with incomparable labels.

Stefan et al. [Stefan et al. 2012] prevent implicit storage channels in their LIO library by assigning static immutable security labels to all mutable shared state. In the context of Trapeze this could be accomplished by statically partitioning the data store into regions with fixed security label While simple and efficient, this solution would not work with existing serverless systems that do not admit such static partitioning, thus defeating our goal of seamless integration with existing serverless software.

We therefore take a different approach to eliminating implicit storage channels that is compatible with unmodified serverless applications. Specifically, we rely on faceted store semantics, where each record can contain several values (facets) with different labels [Austin and Flanagan 2012; Yang et al. 2016]. Facets are created dynamically: when a value with a new label is stored in the record, a facet is created for it (see Section 4 for precise semantics). A read returns the most recent write that is visible to the function. Thus, facets conceal writes with label $b$ from a function running with label $e$, unless $b \sqsubseteq e$.

Example 3.2. We replay the example in Figure 3 with faceted store semantics. Since function labels $b$ and $e$ are incomparable, their respective writes will go into different facets. The functions do not observe each other's writes either explicitly or indirectly, as in Example 3.1.

Faceted stores were previously introduced in IFC research in work on faceted execution [Austin and Flanagan 2012; Austin et al. 2017, 2013; Yang et al. 2016]. The fundamental difference from 
our approach is in the read semantics. Under faceted execution, a read conceptually forks the program, creating a separate branch for each facet read from the store. If the program sends to an external channel, only the branch whose label is compatible with that of the channel is allowed to send. Similar to our design, faceted execution eliminates storage channels; however it does so at a potentially high runtime cost and may become impractical in a system with a large security lattice. In contrast, Trapeze avoids faceted-execution using the apriori knowledge of the function's label by pruning all incompatible facets at read time. Existing faceted execution systems expose the termination channel and therefore enforce TINI, whereas Trapeze enforces the stronger TSNI property.

In the practical use of Trapeze, faceting is an exceptional situation. Trapeze is designed to run unmodified applications that assume conventional store semantics. The moment multiple facets are created in some store location, this semantics is violated, as different functions can now observe different values at the same location. Trapeze treats such situations as attempted exploits and notifies the administrator, who can then take recovery actions, e.g., remove the offending function from the system and rollback the store to the previous consistent state. In the meanwhile Trapeze guarantees that the system continues running without exposing any sensitive information to the attacker.

Faceted store semantics is emulated by the security shim on top of a conventional non-faceted store. In Section 6, we implement facets on top of a key-value store. Yang et al. [Yang et al. 2016] present the design of a faceted SQL database.

Transparency The flip side of Trapeze's strong TSNI security guarantees and light-weight protection is the theoretical loss of transparency, i.e., the ability to run existing unmodified applications. By assigning a static security label to a function, we restrict data that is visible to it. In particular, the function cannot access values above its security level even if it does not send these values (or anything derived from them) through unauthorized channels. On the other hand, all writes to the data store performed by the function are conservatively labeled with the function's label even if they do not carry any secrets.

Both problems can be addressed by refactoring the application. In particular, the function can gain access to secret data via the raiseLabel operation. Conversely, one can avoid tainting data with excessively high labels by splitting the offending function into several functions that run with lower labels. However, if many such changes are required in order to adapt existing applications to work with Trapeze, this will create a barrier to Trapeze's practical adoption.

Our evaluation in Section 6 indicates that in practice the loss of transparency is not an issue in serverless applications due to the common serverless design practice where every function only accesses values that are related to a specific small task and are therefore likely to have compatible security labels.

Covert channels In this work we do not address covert timing channels [Biswas et al. 2017]. A variety of techniques have been proposed for addressing timing channels [Kashyap et al. 2011; Stefan et al. 2012], and incorporating these ideas in Trapeze remains a topic for future work. We do point out that static assignment of security labels to functions complicates timing attacks on Trapeze: since functions cannot observe secret values, such values can not affect their execution time.

At the same time, a faceted store may expose a new timing channel: while a function only observes values that belong to one facet, the presence of multiple facets may affect the timing of data store accesses. However, this channel is hard to exploit in practice: as discussed above, the creation of multiple facets is an exceptional situation in Trapeze, which instantly gets escalated 
to the administrator, who is expected to disable parts of the system under attack or even halt the entire system.

\section{FORMAL SEMANTICS}

In this section, we formalize our IFC semantics for serverless systems with an underlying persistent key-value store. Since computations at different security labels might write to the same key, the key-value store maps each key to a set of values (facets), each with a different label, and we order this set into a sequence according to the temporal order of the writes. The initial store $\sigma_{0}$ maps each key $k$ to the empty sequence: $\sigma_{0}=\lambda k . \epsilon$.

A state $\Sigma \in$ State of the system consists of a key-value store $\sigma \in$ Store, plus a multiset of currently executing serverless function activations called processes (see Figure 4$)$. Each process $p=(t, l)$ consists of a thread $t$ plus its associated security label $l \in$ Label.

Observable events $e \in E v e n t$ of the system include the input event start $p$, which starts a new process $p,{ }^{2}$ and the output event output $c h v$, which sends the value $v$ on output channel $c h$.

The state transition relation $\Sigma \stackrel{e}{\longrightarrow} \Sigma^{\prime}$ describes how the system executes. The first rule [S-START] handles an incoming event start $p$ simply by adding $p$ to the multiset of processes. The next five transition rules all involve executing a particular process $(t, l)$ until its next I/O operation $o p \in$ Operation. For maximal generality, we do not formalize the computation language, but instead assume that the function run: Thread $\rightarrow$ Operation executes the thread $t$ and returns the next $\mathrm{I} / \mathrm{O}$ operation, which includes a continuation for the rest of the thread (analogous to the coinductive definitions used by Bohannon et al. [2009]).

We describe each operation and its corresponding transition rule in turn:

- [s-SEND] for send $\operatorname{ch} v t^{\prime}$ : This rule checks that the process is permitted to output on channel $c h$ (here, label : OutputChannel $\rightarrow$ Label returns the security label of each channel). The process becomes stuck if this check fails. Otherwise, it generates the output event $e=$ output $\operatorname{ch} v$ and the new process state $\left(t^{\prime}, l\right)$ using the continuation $t^{\prime}$ returned from run.

- [S-READ] for read $k f$ : This rule reads the labeled value sequence $\sigma(k)$ from the store; uses the projection operation $\sigma(k) \downarrow_{l}$ defined in Figure 5 to remove all values not visible to the current label; and passes the last entry in this list (i.e. the most recent visible write) to the read continuation $f$.

Note that $\sigma(k) \downarrow_{l}$ may be the empty sequence $\epsilon$, either because key $k$ was never written, or because no such writes are visible to the current process; in this case, last $(\epsilon)$ returns $\perp$, which is passed to $f$.

This rule (and the following four below) generates a dummy observable event nop, since it does not have any externally visible behavior.

- [S-WRITE] for write $k v t^{\prime}$ : In a conventional data store, a new write would overwrite the previous value at that key. In contrast, our faceted store semantics must ensure that a low-label process unable to see the new write will still read an older write. Hence we represent $\sigma(k)$ as a sequence of labeled values. At a new write $(v, l)$ of value $v$ at label $l$, we can garbage-collect or remove all older writes $\left(v^{\prime}, l^{\prime}\right)$ in this sequence $\sigma(k)$ that are no longer visible, namely those where $l \sqsubseteq l^{\prime}$, since any process that could read $\left(v^{\prime}, l^{\prime}\right)$ can also read the more recent write $(v, l)$. The following function performs this garbage collection, and then appends the new labeled value:

\footnotetext{
${ }^{2}$ Serverless systems spawn a new function/process $p$ to handle each incoming event. Here, we assume that each incoming event contains that new process, to simplify the formal development.
} 


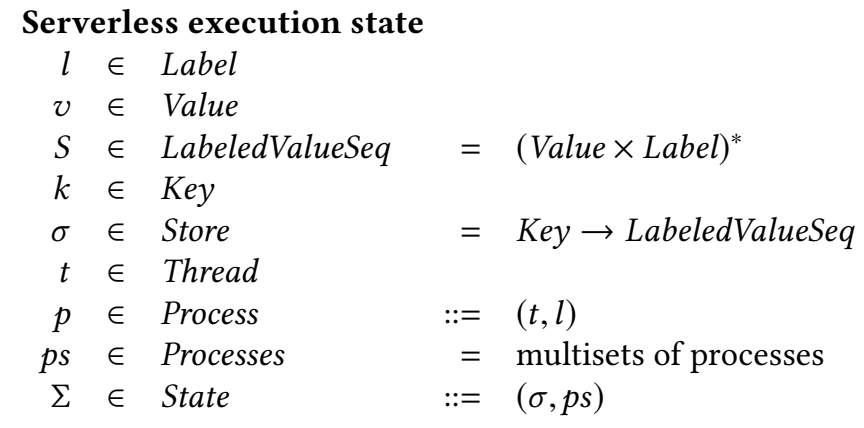

\section{External events and $\mathrm{I} / \mathrm{O}$ operations}

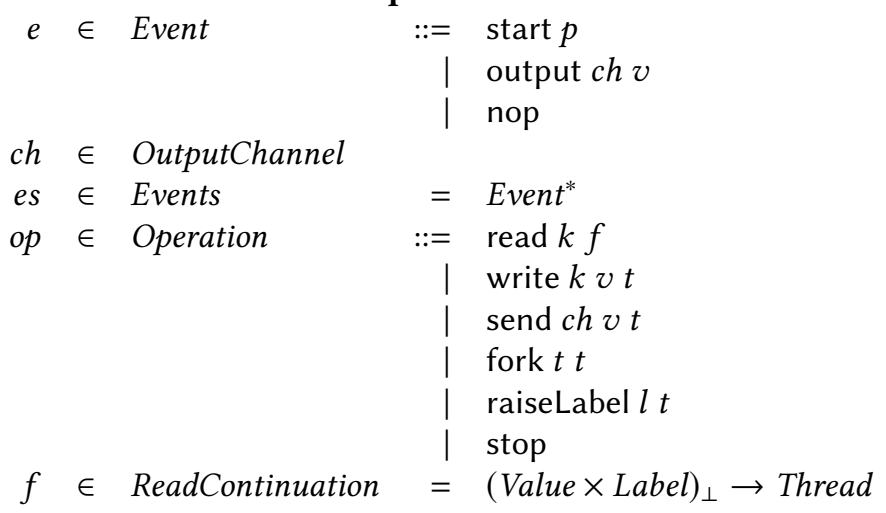

\section{$\sum \stackrel{e}{\longrightarrow} \Sigma$ State transition relation}

\begin{tabular}{|c|c|c|c|c|}
\hline$(\sigma, p s)$ & $\stackrel{\text { start } p}{\longrightarrow}$ & $(\sigma, p s \uplus\{p\})$ & & [S-START] \\
\hline$(\sigma, p s \uplus\{(t, l)\})$ & $\stackrel{\text { tput } \operatorname{ch} v}{\longrightarrow}$ & $\left(\sigma, p s \uplus\left\{\left(t^{\prime}, l\right)\right\}\right)$ & $\begin{array}{l}\operatorname{run}(t)=\operatorname{send} \operatorname{ch} v t^{\prime} \\
l \sqsubseteq \operatorname{label}(\mathrm{ch})\end{array}$ & [S-SEND] \\
\hline$(\sigma, p s \uplus\{(t, l)\})$ & $\stackrel{\text { nop }}{\longrightarrow}$ & $\left(\sigma, p s \uplus\left\{\left(f\left(\operatorname{last}\left(\sigma(k) \downarrow_{l}\right)\right), l\right)\right\}\right)$ & $\operatorname{run}(t)=\operatorname{read} k f$ & [S-READ] \\
\hline$(\sigma, p s \uplus\{(t, l)\})$ & $\stackrel{\text { nop }}{\longrightarrow}$ & $\begin{array}{r}(\sigma[k:=\text { write }(\sigma(k), v, l)], \\
\left.p s \uplus\left\{\left(t^{\prime}, l\right)\right\}\right)\end{array}$ & $\operatorname{run}(t)=$ write $k v t^{\prime}$ & [S-WRITE] \\
\hline$(\sigma, p s \uplus\{(t, l)\})$ & $\stackrel{\text { nop }}{\longrightarrow}$ & $\left(\sigma, p s \uplus\left\{\left(t^{\prime}, l\right)\right\} \uplus\left\{\left(t^{\prime \prime}, l\right)\right\}\right)$ & $\operatorname{run}(t)=$ fork $t^{\prime} t^{\prime \prime}$ & [S-FORK $]$ \\
\hline$(\sigma, p s \uplus\{(t, l)\})$ & nop & $\left(\sigma, p s \uplus\left\{\left(t^{\prime}, l^{\prime}\right)\right\}\right)$ & $\begin{array}{l}r u n(t)=\text { raiseLabel } l^{\prime} t^{\prime} \\
l \sqsubseteq l^{\prime}\end{array}$ & [S-RAISE-LABEL] \\
\hline$(\sigma, p s)$ & $\stackrel{\text { nop }}{\longrightarrow}$ & $(\sigma, p s)$ & & [S-SKIP] \\
\hline
\end{tabular}

$\stackrel{\stackrel{e s}{\longrightarrow}}{\longrightarrow} \Sigma$ Multiple-step state transition relation
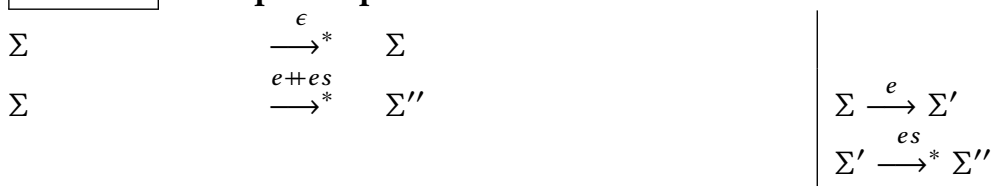

[REFL]

[TRANS]

Fig. 4. Formal semantics. 


$$
\begin{aligned}
S \downarrow_{l} & =S \backslash\left\{\left(v^{\prime}, l^{\prime}\right) \in S \mid l^{\prime} \nsubseteq l\right\} \\
p s \downarrow_{l} & =\operatorname{multiset}\left\{\left(t, l^{\prime}\right) \in p s \mid l^{\prime} \sqsubseteq l\right\} \\
\sigma \downarrow_{l} & =\lambda k \cdot \sigma(k) \downarrow_{l} \\
e \downarrow_{l} & = \begin{cases}e & \exists t, l^{\prime} . e=\operatorname{start}\left(t, l^{\prime}\right) \text { and } l^{\prime} \sqsubseteq l \\
e & \exists c h, v . e=\text { output } \operatorname{ch} v \text { and } \text { label }(\mathrm{ch}) \sqsubseteq l \\
\text { nop } & \text { otherwise. }\end{cases} \\
\left(e_{1} \ldots e_{n}\right) \downarrow_{l} & =e_{1} \downarrow_{l} \ldots e_{n} \downarrow_{l} \\
(\sigma, p s) \downarrow_{l} & =\left(\sigma \downarrow_{l}, p s \downarrow_{l}\right)
\end{aligned}
$$

Fig. 5. Definition of projection function.

$$
\begin{aligned}
& \text { write }: \text { LabeledValueSeq } \times \text { Value } \times \text { Label } \rightarrow \\
& \text { LabeledValueSeq } \\
& \text { write }(S, v, l)=\left(S \backslash\left\{\left(v^{\prime}, l^{\prime}\right) \in S \mid l \sqsubseteq l^{\prime}\right\}\right)+(v, l)
\end{aligned}
$$

The symbol + denotes sequence concatenation.

- [P-FORK] for fork $t^{\prime} t^{\prime \prime}$ : This rule forks a new thread $t^{\prime \prime}$, where $t^{\prime}$ is the continuation of the original thread. Both threads inherit the security label of the original process.

- [s-RAISE-LABEL] for raiseLabel $l^{\prime} t^{\prime}$ : This rule simply raises the label of the current process to a higher label $l^{\prime}$, which, for example, permits the process to read more secret data.

- Finally, [s-SKIP] allows a state to perform stuttering nop steps at any time, as a technical device to facilitate the non-interference proof below.

Note that no rule is needed for the stop operation. Instead we just leave the stopped processes in the process multiset for simplicity.

\section{TERMINATION SENSITIVE NON-INTERFERENCE}

We use the notation $\bullet \downarrow_{l}$ on various domains to remove any information that is not visible to an observer at level $l$ (see Figure 5). For example: $S \downarrow_{l}$ contains only values with labels visible to $l$, and $p s \downarrow_{l}$ contains only processes with labels visible to $l$. An event $e$ is visible to $l$ if it starts a process visible to $l$, or if it outputs on a channel visible to $l$; otherwise, we say $e \downarrow_{l}=$ nop. We write $\bullet_{1} \approx_{l} \bullet_{2}$ to denote that items appear equivalent to an observer at level $l$, i.e., $\bullet_{1} \downarrow_{l}=\bullet_{2} \downarrow_{l}$.

Our proof is based on the projection lemma below, which relates the execution of the full system $\Sigma$ and the portion $\Sigma \downarrow_{l}$ visible at level $l$. Every step of $\Sigma$ has a corresponding step in $\Sigma \downarrow_{l}$ (part 1) and vice versa (part 2).

\section{Lemma (Projection).}

Part 1. If

$$
\Sigma \stackrel{e_{1}}{\longrightarrow} \Sigma_{1}^{\prime}
$$

then for some $\Sigma_{2}^{\prime}$ and $e_{2}$,

$$
\Sigma \downarrow_{l} \stackrel{e_{2}}{\longrightarrow} \Sigma_{2}^{\prime} \quad \Sigma_{1}^{\prime} \approx_{l} \Sigma_{2}^{\prime} \quad e_{1}^{\prime} \approx_{l} e_{2}^{\prime}
$$

Part 2. If

$$
\Sigma \downarrow_{l} \stackrel{e_{1}}{\longrightarrow} \Sigma_{1}
$$

then for some $\Sigma_{2}^{\prime}$ and $e_{2}$,

$$
\Sigma \stackrel{e_{2}}{\longrightarrow} \Sigma_{2} \quad \Sigma_{1} \approx_{l} \Sigma_{2} \quad e_{1} \approx_{l} e_{2}
$$

Proof. See appendix. 
Based on this lemma, our proof of single-step and multi-step termination sensitive noninterference is straightforward. ${ }^{3}$

\section{Theorem (Single step Termination Sensitive Non-Interference).} If

then for some $\Sigma_{2}^{\prime}$ and $e_{2}$,

$$
\Sigma_{1} \approx_{l} \Sigma_{2} \quad \Sigma_{1} \stackrel{e_{1}}{\longrightarrow} \Sigma_{1}^{\prime}
$$

Proof. By Projection (Part 1):

$$
\Sigma_{2} \stackrel{e_{2}}{\longrightarrow} \Sigma_{2}^{\prime} \quad \Sigma_{1}^{\prime} \approx_{l} \Sigma_{2}^{\prime} \quad e_{1} \approx_{l} e_{2}
$$

$$
\Sigma_{1} \downarrow_{l} \stackrel{e_{3}}{\longrightarrow} \Sigma_{3}^{\prime} \quad \Sigma_{1}^{\prime} \approx_{l} \Sigma_{3}^{\prime} \quad e_{1} \approx_{l} e_{3}
$$

for some $\Sigma_{3}^{\prime}$ and $e_{3}$. The $l$-equivalence assumption implies that $\Sigma_{2} \downarrow_{l} \stackrel{e_{3}}{\longrightarrow} \Sigma_{3}^{\prime}$. By Projection (Part 2):

$$
\Sigma_{2} \stackrel{e_{2}}{\longrightarrow} \Sigma_{2}^{\prime} \quad \Sigma_{3}^{\prime} \approx_{l} \Sigma_{2}^{\prime} \quad e_{3} \approx_{l} e_{2}
$$

for some $\Sigma_{2}^{\prime}$ and $e_{2}$. Therefore, by transitivity of $\approx_{l}$, we have $\Sigma_{1}^{\prime} \approx_{l} \Sigma_{2}^{\prime}$ and $e_{1} \approx_{l} e_{2}$, as required.

\section{Corollary (Termination Sensitive Non-Interference). \\ If}

$$
\Sigma_{1} \approx_{l} \Sigma_{2} \quad \Sigma_{1} \stackrel{e s_{1}}{\longrightarrow} \Sigma_{1}^{\prime}
$$

then for some $\Sigma_{2}^{\prime}$ and $e_{2}$,

$$
\Sigma_{2} \stackrel{e s_{2}}{\longrightarrow} \Sigma_{2}^{\prime} \quad \Sigma_{1}^{\prime} \approx_{l} \Sigma_{2}^{\prime} \quad e s_{1} \approx_{l} e s_{2} .
$$

Proof. By induction on the derivation of $\Sigma_{1} \stackrel{e s_{1}}{\longrightarrow} \Sigma_{1}^{\prime}$.

Our Coq formalization of these semantics and proofs is available at https://github.com/kalevalp/ trapeze/blob/master/coq-proof/proof.v.

Our TSNI result states that the set of observable outputs of the system under all possible schedules does not depend on inputs that are not visible to the observer. This result does not prevent a malicious scheduler from leaking secrets by prioritizing certain schedules, e.g., by scheduling low-security processes based on high-security secrets. However, as mentioned earlier, we assume that the scheduler is not adversarial in this manner. Prior work [Stefan et al. 2012] has addressed this problem by assuming a round-robin scheduler, but this assumption is not realistic for serverless computing.

\section{IMPLEMENTATION AND EVALUATION}

\subsection{Implementation}

In order to evaluate our proposed security architecture and IFC model, we developed an open-source prototype implementation of Trapeze [Alpernas et al. 2017]. The implementation is portable and currently runs on the two most popular serverless platforms-AWS Lambda [Amazon 2017a] and OpenWhisk [Apache Software Foundation 2017]. It consists of three components: the sandbox, the security shim, and the authentication service.

Sandbox The Trapeze sandbox encapsulates application code, redirecting all its inputs and outputs through the security shim (Figure 1). The exact sandboxing technology depends on the programming language used. We currently support serverless functions written in JavaScript for the node.js runtime, which is one of the most common types of serverless functions on both AWS and IBM Cloud Functions, IBM's public OpenWhisk service.

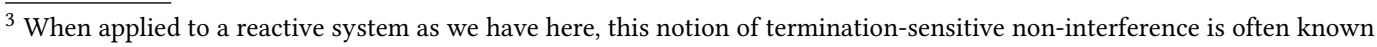
as progress-sensitive non-interference.
} 
We encapsulate node.js functions using the VM2 sandbox [VM2 2017]. VM2 relies on proxy objects [Van Cutsem and Miller 2013] to restrict untrusted JavaScript code to only interact with the world outside of the sandbox via whitelisted interfaces. Specifically, we restrict the serverless function inside the sandbox to only be able to invoke methods of the security shim. By design, the VM2 sandbox cannot be bypassed by buggy or malicious sandboxed code.

The use of a language-based sandbox technology means that both the sandbox and the JavaScript runtime are part of the Trapeze TCB. For example, bugs in low-level $\mathrm{C} / \mathrm{C}++$ bindings used by the JavaScript runtime [Brown et al. 2017] can in principle be exploited to break out of the sandbox. Brown et al. [2017] propose an efficient static analysis to detect such bugs.

Restricting access to local state Our IFC model assumes that a function starts from a clean state and can only observe secrets passed to it through arguments or read from the shared store. In practice, serverless platforms expose other types of state, access to which must be restricted to maintain the TSNI guarantee. In particular, serverless functions typically run in a container environment. For efficiency, the container and the node.js process that executes the function are reused across multiple invocations. This allows functions to cache local state inside the container There are two types of such persistent container-local state: (1) global variables inside the node.js process, (2) external state stored in the local file system and processes.

Our implementation restricts the use of both types of local state, enforcing IFC model invariants. First, our sandbox re-initializes the encapsulated node.js process to clean state on each serverless invocation, thus preventing state sharing through global variables. Note that this behavior is compatible with the standard serverless semantics, which does not guarantee that node.js state is preserved across invocations.

Second, our sandbox configuration by default blocks accesses to all OS resources, including files and processes. To support existing serverless applications that use these resources, we would like to re-enable access to selected resources in a secure way. To this end, we implement adapters that enforce IFC semantics for specific resource types. We have so far implemented an adapter for securely running external processes (described below). Support for other resources can be added in the future. For example, we envisage a file system adapter that enforces faceted semantics for local file accesses.

Security shim The security shim monitors all inputs and outputs of a function and enforces IFC rules. The shim consists of multiple adapter modules, one for each supported input and output interface. There are four groups of adapters: (1) data store adapters, (2) function call adapters, (3) external channel adapters, (4) local state adapters.

A data store adapter implements faceted store semantics on top of a conventional cloud data store. Trapeze currently supports a single type of data store-a faceted key-value store implemented on top of a relational database. The key-value store implements a standard dictionary with the following operations: put(key, value), get(key), del(key), and keys() (returns all keys in the store). The store is backed by a relational database table with 3 columns, for the key, value, and label. The table contains an entry for each facet of each value in the store. We used MySQL server available in AWS through Amazon Relational Database Service. The security shim passes an additional parameter to every operation-the security label. The get operation performs an SQL query that returns all entries that match the given key, and whose label is less than or equal to the given label. The del operation deletes all facets with labels greater than or equal to the given label The put operation deletes the same elements a del does, and then inserts the given key-value pair, with the given label. In addition to the faceted version, we also provide a conventional insecure key-value store implementation as a baseline for performance evaluation. 
Function call adapters support different ways to invoke serverless functions, making sure that the callee inherits the caller's label, as required by our IFC model. We support two invocation mechanisms: AWS Step Functions, which run a workflow with multiple serverless functions, controlled by a finite automaton, and Amazon Kinesis, which supports asynchronous communication via real-time event streams.

External channel adapters enable secure communication across the cloud boundary. The supported types of channels are (1) user-initiated HTTP sessions, (2) email communication via the Nodemailer module [nodemailer 2017], and (3) connections to external S3 buckets (used to upload large data objects that do not fit in HTTP requests). HTTP sessions obtain their security labels from the authentication service (see below). The Nodemailer adapter uses the user database (see below) to map an email address to a user security label. The S3 adapter inherits the label of the user who provides login credentials for the S3 bucket.

Local state adapters mediate access to local OS resources such as files and processes, as discussed above. For instance, many serverless functions invoke external programs. We would like to allow such functionality in Trapeze, while enforcing that the external program cannot leak secrets to the outside world. To this end, we implemented an adapter that runs arbitrary programs in a ptrace-based sandbox [Fouladi et al. 2017a]. The sandbox restricts the program's I/O activity to a temporary local directory, which gets purged on every serverless function invocation.

Authentication service The authentication service is responsible for associating a correct security label with every external HTTP session. It is implemented on top of a user database that stores credentials, email addresses, and security labels of all users in the system.

The entire Trapeze framework consists of 1174 lines of JavaScript code, including 649 lines in the the AWS and OpenWhisk shim modules, 484 lines in the key-value store, and 41 lines in the authentication service.

\subsection{Declassifiers}

Many real-world applications allow limited flow of information down the security lattice. For example, a credit reporting agency may make the distribution of consumers across credit score bands publicly available. This statistics is computed based on the credit history of all consumers and must therefore be labeled with the least upper bound of all their labels. However, since the aggregate statistics exposes negligible amount of information about individual consumers, it can be safely declassified.

Similar to previous IFC models, Trapeze introduces declassifiers to support such scenarios. A declassifier is a triple $\langle h, l, D\rangle$, where $h$ and $l$ are security labels, such that $l \sqsubset h$, and $D$ is a serverless function. A declassifier is invoked just like any other serverless function; however its security label is computed using special rules. Let $x$ be the label of the calling function. Then the declassifier is assigned label $x^{\prime}$, such that:

$$
x^{\prime}= \begin{cases}l, & \text { if } l \sqsubseteq x \sqsubseteq h \\ x, & \text { otherwise }\end{cases}
$$

By design, declassifiers violate the non-interference property; therefore the formal model and proofs in Section 4 are given for the pure IFC model without declassifiers.

\subsection{Evaluation Questions}

Our evaluation aims to answer the following questions:

(1) Security: Can Trapeze enforce information security in real-world serverless applications? In particular, can confidentiality requirements of such applications be captured in a security 


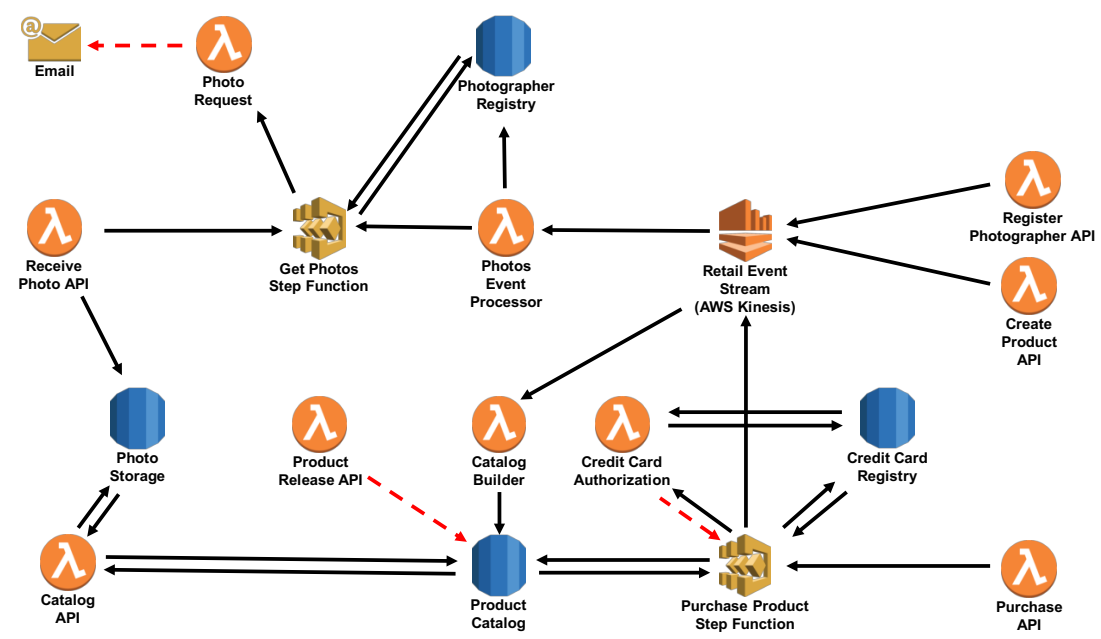

Fig. 6. The architecture of the Hello, Retail! project. Circles labeled $\lambda$ represent the main functions of this application. Functions whose names end with 'API' can be directly invoked via client HTTP requests. Arrows show interactions between different components. Red dashed arrows indicate interactions that carry declassified data.

policy consisting of a security lattice and trusted declassifiers? Can Trapeze enforce the policy in the presence of buggy or malicious code?

(2) Transparency: Can Trapeze secure existing serverless applications with minimal modifications?

(3) Performance: Can Trapeze achieve the first two goals with low performance overhead?

\subsection{Case Studies}

To answer the above questions, we carried out three case studies where we used Trapeze to add a security layer to existing serverless applications. We outline each of the case studies below.

Case study 1: Hello, Retail! Hello, Retail! is a project from the serverless team at Nordstrom. The goal was to produce a purely serverless, back-end for an e-commerce web site. It has since been open-sourced [Nordstrom Technology 2017] and won the architecture competition award at serverlessConf Austin'17 [McKim, John 2017].

We made several changes to Hello, Retail! before applying Trapeze to it. First, we replaced DynamoDB and S3 databases in Hello, Retail! with with calls to Trapeze's key-value store. Second, we replaced calls to the Twilio SMS messaging service, which is currently not supported by Trapeze, with e-mail communication. Third, we extended the Hello, Retail! project with a product purchase subsystem, which manages online orders and credit card payments. The resulting system consists of 21 serverless functions. Figure 6 shows the high-level architecture of the system.

The system serves several types of users: (1) the store owner, who manages the online catalog and processes orders, (2) photographers, who upload product images to the catalog, (3) customers, who navigate the catalog and place orders, and (4) the VISA credit card authority, which authorizes card payments on behalf of customers. The security lattice (Figure 7) consists of labels, matching these user categories. Solid lines in the diagram show the partial order of security labels; dashed arrows show declassifiers, with a declassifier $\langle h, l, D\rangle$ represented by an arrow from label $h$ to $l$. Table 1 summarizes the security labels in this case study. 
Table 1. Security classes in the Hello, Retail! case study.

\begin{tabular}{|l|l|}
\hline label & description \\
\hline \hline owner & $\begin{array}{l}\text { Sensitive information managed by the store owner, including product catalog } \\
\text { and photographers' email addresses. Items in the catalog are only visible to } \\
\text { the owner until they are released to the public via the release declassifier. } \\
\text { Labels online purchases of a specific customer. This information is visible to } \\
\text { the customer and the store owner (since } \text { client }_{j} \sqsubset \text { owner) }\end{array}$ \\
clientCC $j$ & $\begin{array}{l}\text { Customer credit card information that can only be released to a credit card } \\
\text { authority } \\
\text { Labels the external communication channel to the VISA credit card authority } \\
\text { VISA }\end{array}$ \\
photographer $_{i}$ & Product photos uploaded by a photographer \\
\hline
\end{tabular}

The following scenario illustrates the flow of sensitive information through the Hello, Retail! system. Every step in the scenario is annotated with security label(s) of data involved in this step.

1. [owner] The owner creates a new product description in the catalog.

2. [photographer ${ }_{i}$ ] The owner sends an email to one of the photographers requesting a picture

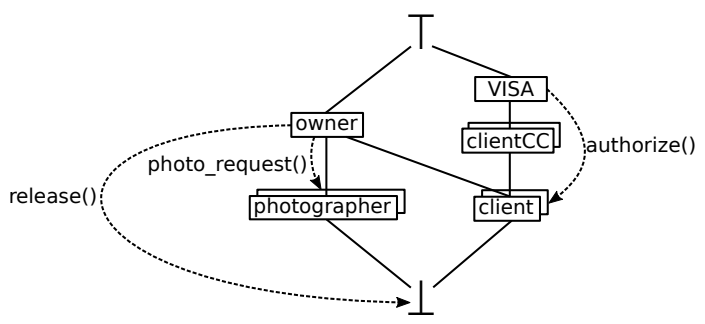

Fig. 7. Security lattice and declassifiers in the Hello, Retail! case study. of the product. The request includes information from the product description, declassified with the 〈owner, photographer ${ }_{i}$, photo_request ()$\rangle$ declassifier, which implements a trusted user interface that request owner's confirmation of the declassification.

3. [ photographer $_{i}$ ] The photographer uploads a product image to the catalog.

4. $[\perp]$ Once the owner is ready to make the product publicly available in the online catalog, she declassifies it using the $\langle$ owner, $\perp$, release ()$\rangle$ declassifier.

5. $\left[\right.$ client $_{j} /$ client $\left.C_{j}\right]$ A client orders a product from the catalog. Order information, labeled client $_{j}$ is visible to the the client as well as the owner, since client $_{j} \sqsubset$ owner. Client's credit card details

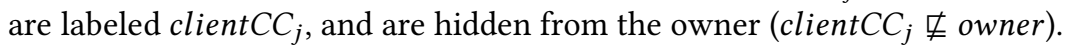

6. [VISA] Before the order is finalized, credit card information is sent to VISA for payment authorization through an external channel labeled VISA.

7. $\left[\right.$ client $\left._{j}\right]$ The response received through this channel consists of one bit of information indicating success or failure, which gets declassified by the $\left\langle\right.$ VISA, client $_{j}$, authorize ()$\rangle$ declassifier, making the outcome of the request visible to the client and the owner.

Case study 2: gg gg [Fouladi et al. 2017a; gg project 2017] is a system for running parallel software workflows, such as software compilation and video processing, on serverless platforms. In gg, each unit of work, or thunk, specifies both the executable to run and all its data dependencies. The workflow is synthesized as a direct acyclic graph (DAG) of thunks, and is recursively executed on a serverless platform by gg's execution engine. gg identifies each dependency in a content-addressed way, using a key-value store as the storage backend. gg consists of a single serverless function, which internally runs arbitrary user-provided executables. Each invocation of the function executes exactly one thunk by fetching the dependencies from the object store, executing the thunk, and storing the output back into the object store. 
We use a parallel build framework implemented on top of gg [Fouladi et al. 2017a] as a concrete use case. The framework extracts a workflow DAG, where every thunk corresponds to an invocation of a build tool (e.g., a compiler or a linker), from the project makefile.

The original gg implementation is single-tenant, with every authenticated user having access to all sources and binaries in the system. We use Trapeze to introduce a secure multi-tenant mode to gg. In this mode, tenants only have access to their own source and compiled code. A thunk running on behalf of the tenant taints all of its outputs with the tenant's label. A tenant may release some of their sources or compiled binaries to the public, making them available to all other tenants. This is reflected in the security lattice in Figure 8 with mutually incomparable tenant labels

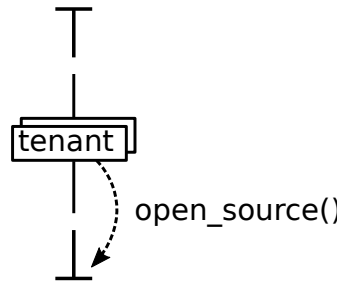

Fig. 8. gg security lattice. and a $\left\langle\right.$ user $_{i}, \perp$, open_source ()$\rangle$ declassifier.

Prior to adding the multi-tenant mode to gg, we ported parts of it that were written in Python to JavaScript, as well as modified it to use our key-value store.

Case study 3: Image feature extraction This serverless application gives its users access to Amazon's AWS Rekognition image analysis service [Amazon 2017b]. It is based on the 'Fetch File and Store in S3' and 'Analyse Image from S3' examples from the Serverless Examples collection [Serverless, Inc 2017].

The application consists of the upload function that takes an image URL, fetches the image and stores it in the key-value store, and the feature extraction function that uses AWS Rekognition to extract features from the image and send them to the user.

We use Trapeze to add a security layer to this example, enforcing that every user can only access information extracted from images they own. This policy is expressed in the simple security lattice in Figure 9.

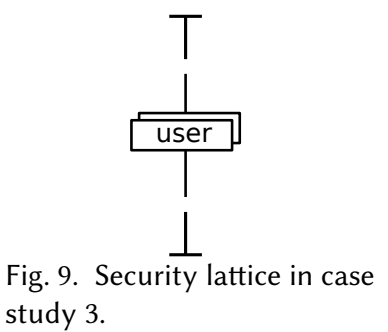

\subsection{Security}

We employ Trapeze to protect sensitive data in the three case studies. The original implementation of these applications either offered coarse-grained protection, giving every authenticated user access to all data in the system, or implemented ad hoc security policies embedded in application code. For example, the Hello, Retail! system by design only exposes credit card details to the credit card authority. Such protection relies on checks scattered around the application code and is easy to get wrong. Besides, it can be bypassed by any exploit that subverts the application logic.

Trapeze captures the security requirements of each of the three case studies in a security policy consisting of a security lattice and declassifiers, shown in Figures 7, 8, 9. Our policies are simple and concise, consisting of only several classes of labels and few declassifiers. The policies are decoupled from the application logic and its software architecture. For instance, adding new functions to the application, changing its control flow, or even refactoring the database schema, do not affect the security policy.

Furthermore, Trapeze is immune to malicious or compromised application logic. We simulated code injection attacks in our case studies by replacing some of the original functions with malicious functions that attempt to leak secrets to unauthorized users, similar to examples in Figures $2 \mathrm{~b}$ and 3. As expected, these simulated attacks failed when running the application with Trapeze. 
Table 2. Size of the case studies in lines of code (LoCs): (1) LoCs comprising the application, (2) lines added or modified to adapt the application to work with Trapeze, (3) LoCs in trusted declassifiers.

\begin{tabular}{|l|c|c|c|}
\hline case study & app code (LoCs) & modif. code (LoCs) & declassifier code (LoCs) \\
\hline \hline Hello, Retail! & 1,300 & 3 & 104 \\
gg & 8,800 & 2 & 94 \\
Feature Extraction & 95 & 0 & 0 \\
\hline
\end{tabular}

\subsection{Transparency}

As discussed in Section 3, Trapeze enforces TSNI at the cost of reduced transparency, which may require the developer to refactor the application to work with Trapeze.

Table 2 measures the loss of transparency in our case studies by reporting the size of changes to application code in terms of lines of code (LoCs) required to adapt the application to work with Trapeze. This does not take into account the changes needed to port the application to use our key-value store as well as other compatibility changes that are not fundamental to the Trapeze architecture and can be made unnecessary with some additional engineering effort.

None of the case studies required splitting a function into multiple functions. Hello, Retail! required calling raiseLabel twice, once in order to upgrade the label of the purchase placement function from client $_{j}$ to client $C C_{j}$ before saving the customer's credit card details in the data store, and once to upgrade the label of the payment authorization function from client $_{j}$ to VISA, in order to read credit card details from the store and send them to the credit card authority. In addition, the gg case study required a minor change due to a technicality: the existing code was not fully compatible with the VM2 sandbox.

These results indicate that in practice the loss of transparency is not an issue in serverless applications. This is due to the common software design practices in the serverless world, where each function is assigned a single small task and only accesses the values that are related to this task and are therefore likely to have compatible security labels.

The last column of the table reports the total size of declassifiers used in each case study. This number characterizes the amount of trusted application code in each example.

\subsection{Performance}

We measure the overhead of Trapeze by running case studies 1 and 3 on the AWS Lambda serverless platform. We run case study 2 on the OpenWhisk platform in the IBM Cloud (formerly, IBM Bluemix), since AWS Lambda does not support ptrace, which we use to sandbox binary executables in gg (Section 6.1).

Table 3 summarizes the runtime overhead of Trapeze. Since the overhead may depend on the exact workload, we constructed several typical workflows for each case study. We test the Hello, Retail! case study on two workloads: the 'Build\&Browse' workload, that simulates the construction and browsing of the product catalog, and the 'Update\&Purchase' workload, that simulates updates to the catalog followed by a series of online purchases. We test gg by using it to compile four open source software packages: mosh, git, vim, and openssh. Finally, we consider two scenarios for the Image Feature Extraction: the 'Image Upload' scenario, that uploads a single image to the data store, and the 'Feature Extraction' scenario that performs feature extraction for a stored image.

For each workload, we report the total number of serverless function calls (the $\# \lambda$ column in Table 3). The 'function runtime' section of the table reports the total runtime of all functions in the scenario with and without protection and the relative slowdown introduced by protection, averaged across 10 runs. These runtimes do not include additional declassifier calls introduced in 
Table 3. Trapeze performance.

\begin{tabular}{|l|c|c|c|c|}
\hline \multirow{2}{*}{ scenario } & \multirow{4}{*}{$\# \lambda$} & function runtime (ms) & \multirow{2}{*}{$\Delta(\%)$} \\
\cline { 2 - 4 } & & insecure & Trapeze & \\
\hline \hline \multicolumn{5}{|c|}{ Hello, Retail! } \\
\hline Update\&Purchase & 435 & 51,246 & 98,563 & 92.33 \\
Build\&Browse & 955 & 257,799 & 391,900 & 52.03 \\
\hline \multicolumn{5}{|c|}{ gg } \\
\hline mosh & 111 & 681,173 & 654,448 & -3.92 \\
git & 917 & $2,602,500$ & $2,660,721$ & 2.24 \\
vim & 234 & $1,242,873$ & $1,338,128$ & 7.66 \\
openssh & 654 & $1,626,223$ & $1,649,139$ & 1.41 \\
\hline \multicolumn{4}{|c|}{ Image Feature Extraction } \\
\hline Image Upload & 1 & 475 & 525 & 10.4 \\
Feature Extraction & 1 & 1,882 & 2,114 & 12.3 \\
\hline
\end{tabular}

Table 4. Storage overhead of Trapeze.

\begin{tabular}{|l|c|c|c|}
\hline case study & insecure (KB) & Trapeze (KB) & $\Delta(\%)$ \\
\hline \hline Hello, Retail! & 2,704 & 3,200 & 18.34 \\
gg & $15,054,000$ & $15,118,496$ & 0.43 \\
Feature Extraction & 8,256 & 8,416 & 1.94 \\
\hline
\end{tabular}

the protected execution. Accounting for declassifiers increased the total runtime by another $10 \%$ in the Build\&Browse workload, while not making any measurable impact on other workloads. In our initial experiments we also measured the total time to execute each workload; however we found that, due to the non-deterministic nature and varying resource availability in the serverless environment, these times varied wildly across different runs and did not provide any insights into the performance of Trapeze.

For most workloads, Trapeze adds a modest overhead of up to $12.3 \%$ to function runtime. The negative overhead in the gg mosh workload is due to the noisy IBM Cloud environment, where the runtime of a function varies dramatically across different invocations based on the load on the node where the function is scheduled. The overhead is higher in the Hello, Retail! case study, up to $92.33 \%$ in the Update\&Purchase scenario. Further benchmarking revealed that the bulk of this overhead is due to the startup time of the VM2 sandbox, which adds $100 \mathrm{~ms}$ on average per function invocation. Since all functions in this example have short runtimes, the startup time becomes a significant contributor to the total runtime. We measured that two thirds of the startup time is spent loading libraries used by the application. In Section 6.8 we present an approach that eliminates most of this overhead.

Finally, we evaluate the storage overhead of our secure key-value store. Table 4 compares database sizes (in kilobytes) for an insecure and secure versions of the store for each of the three case studies (we report results for one of the workloads in each case study, as the relative increase in the database size is independent of the workload). The secure database requires more space, as it stores a security label with each value. This overhead is low (under $2 \%$ ) in examples where the database stores large objects, e.g., images or source files. It is more significant (18.34\%) in the Hello, Retail! case study where individual values stored in the database are small. 
Table 5. Overhead results of the fork optimized Trapeze.

\begin{tabular}{|c|c|c|c|c|c|}
\hline & \multicolumn{3}{|c|}{ average function runtime (ms) } & \multicolumn{2}{c|}{$\Delta(\%)$} \\
\cline { 2 - 6 } & insecure & Trapeze & fork optimized Trapeze & Trapeze & fork-opt \\
\hline \hline Microbenchmark 1 & $126.21 \mathrm{~ms}$ & $274.29 \mathrm{~ms}$ & $154.26 \mathrm{~ms}$ & $117.31 \%$ & $22.23 \%$ \\
Microbenchmark 2 & $226.82 \mathrm{~ms}$ & $354.85 \mathrm{~ms}$ & $250.31 \mathrm{~ms}$ & $56.45 \%$ & $10.36 \%$ \\
\hline
\end{tabular}

\subsection{Recovering the Warm Start Optimization in Trapeze}

A bulk of the overhead observed in the Hello, Retail! case study is due to the loss of the warm start optimization with Trapeze. Serverless platforms load the execution environment once per function instance and reuse it across multiple invocations. In case of node.js functions, this entails pre-loading JavaScript modules required by the function. The resulting closure may be used to persist global variables within these modules across invocations, thereby bypassing the security mechanism. As discussed in Section 6.1, we prevent this by discarding the sandbox along with all global state on function termination. This results in a secure execution, at the cost of running all module-loading and environment-setup code on every function invocation.

Ideally, we would like to reuse pre-loaded libraries across multiple invocations without exposing changes to the global state. We achieve this using the following optimization that exploits the POSIX fork system call. When a function is instantiated inside a container, we pre-load all JavaScript libraries inside the sandbox and fork the node.js process. We execute the serverless function in the child process, terminating the process upon function completion. On each subsequent invocation, the parent process forks a new child. The child inherits a fully initialized environment, but since it runs in a separate address space, the execution of the function within the child process does not affect the parent process, ensuring that subsequent invocations of the function are unaffected by any changes made by previous invocations.

fork calls are substantially faster than module loads. In our experiments, fork calls took at most $1 \mathrm{~ms}$. Subsequently, this approach results in a substantial recovery of performance, while retaining the same security guarantees.

In order to evaluate the performance gains from the fork optimization, we have measured the running times of two microbenchmarks that simulate the overheads seen in the Hello, Retail! case study. The microbenchmarks load the same modules and configuration files that are loaded in the functions of the Hello, Retail! case study, and idle for 125ms and 225ms in Microbenchmark 1 and Microbenchmark 2 respectively, to simulate computations performed by Hello, Retail! functions. The fork optimized version of Trapeze showed significantly improved overheads, in one case reducing the overhead from $56 \%$ in the original Trapeze implementation to $10 \%$ in the fork optimized version, and in the other reducing the overhead from $117 \%$ to $22 \%$. The results are summarized in Table 5.

Unfortunately, the fork optimization is not immediately applicable to the Hello, Retail! case study. The Kinesis stream API used in Hello, Retail! does not work correctly when invoked from a forked process. This is not a fundamental limitation, but it requires support from the Amazon AWS team to fix. However, based on our microbenchmarks, we are confident that a low-overhead solution can be implemented based on the foundations provided by Trapeze.

\section{RELATED WORK}

The modern technique for using security labels to dynamically monitor information flow was proposed by Denning [1976] as the Lattice Model. That work also defines the concept of implicit flow of information. Austin et al. [2017] describe three techniques for monitoring such flows, namely 
Failure Oblivious and No Sensitive Upgrade and Permissive Upgrade. Our work exposes a fourth choice using faceted values specifically to eliminate implicit flows.

Austin and Flanagan [2012] introduce faceted values as a full language-based enforcement technique: rather than focusing specifically on controlling implicit flows, they combine the faceted value concept with the concept of multiple executions, which enables more precise enforcement at the cost of more runtime overhead.

Trapeze enforces a strong form of non-interference, TSNI. Most existing IFC systems support only termination-insensitive non-interference (TINI). The few previous systems that enforce TSNI are either too restrictive or too costly for most practical applications. The multilevel security (MLS) model [Bell and LaPadula 1973] achieves TSNI by statically partitioning all the code and data in the system into security compartments. This model, designed primarily for military-grade systems, is too restrictive for most applications and requires complete re-design of existing software. Smith and Volpano [Smith and Volpano 1998] present a security type system that enforces TSNI by imposing a harsh restriction that loop conditions may not depend on secret data. Heintze and Riecke [Heintze and Riecke 1998] propose a secure typed Lambda calculus called SLam. While SLam only enforces TINI, Sabelfeld and Sands [Sabelfeld and Sands 2001] point out that a version of SLam with lazy evaluation semantics would be termination-sensitive.

Indeed, Stefan et al. [2012] implemented a Haskell library called LIO, which guarantees TSNI by requiring programmers to decompose their programs into separate threads with floating labels These are analogous to the processes in our formalism. LIO prevents implicit storage channels by assigning immutable security labels to state shared between processes. In contrast, Trapeze uses faceted values instead.

Secure multi-execution [Devriese and Piessens 2010] achieves TSNI by running multiple independent copies of the program, one for each security class. This technique introduces CPU and memory overhead proportional to the number of security classes. While acceptable in systems with few security classes (e.g., Devriese and Piessens [Devriese and Piessens 2010] consider only two classes, secret and non-secret), this becomes impractical in cloud-scale systems with potentially millions of mutually untrusting users. Faceted execution has the potential to mitigate this drawback, and Bielova and Rezk [2016] have proposed a theoretical approach for extending the faceted execution model to enforce TSNI.

The Asbestos OS [Efstathopoulos et al. 2005] applies dynamic IFC at the granularity of an OS process, similar to how Trapeze operates at the granularity of a serverless function. Asbestos associates a static security label with each process; however this label only serves as an upper bound on the label of data the process can access. Process's effective label changes dynamically, which enables the implicit termination channel.

To the best of our knowledge, Trapeze is the first system to apply IFC to serverless applications. Several researchers advocate the use of IFC in the broader context of secure cloud computing [Bacon et al. 2014; Pasquier et al. 2016]; however we are not aware of a practical implementation of these ideas.

\section{CONCLUSION}

The advent of serverless computing provides the opportunity to rebuild our cloud computing infrastructure based on a rigorous foundation for information flow security. We present a novel and promising approach for dynamic IFC in serverless systems. This approach combines (1) a sandbox and security shim that monitors all I/O operations of each serverless function invocation; (2) static security labels for each serverless function invocation; and (3) dynamic faceted labeling of data in the persistent store. 
This combination of ideas provides the strong security guarantee of TSNI, which is necessary in serverless settings to avoid high-bandwidth termination channel leaks via multiple concurrent requests.

Our Trapeze implementation of this approach is lightweight, requiring no new programming languages, compilers, or virtual machines. The three case studies show that Trapeze can enforce important IFC properties with low space and time overheads.

We believe Trapeze represents a promising approach for deploying serverless systems with rigorous security guarantees that help prevent costly information leaks arising from buggy or mis-configured application code or from code-injection attacks.

\section{A SUMMARY OF AUXILIARY SEMANTIC DETAILS}

$\epsilon$ is the empty sequence and + is concatenation.

label : OutputChannel $\rightarrow$ Label

run : Thread $\rightarrow$ Operation

write : LabeledValueSeq $\times$ Value $\times$ Label $\rightarrow$

LabeledValueSeq

write $(S, v, l)=\left(S \backslash\left\{\left(v^{\prime}, l^{\prime}\right) \in S \mid l \sqsubseteq l^{\prime}\right\}\right)+(v, l)$

$\bullet_{1} \approx_{l} \bullet_{2}$ means $\bullet_{1} \downarrow_{l}=\bullet_{2} \downarrow_{l}$

\section{B PROOF DETAILS}

\section{Lemma (Invisibility).}

If

$$
\begin{gathered}
l^{\prime} \nsubseteq l \\
\left(\sigma, p s \uplus\left\{\left(t, l^{\prime}\right)\right\}\right) \stackrel{e}{\longrightarrow}\left(\sigma^{\prime}, p s \uplus p s^{\prime}\right)
\end{gathered}
$$

then

$$
\begin{aligned}
\sigma^{\prime} \downarrow_{l} & =\sigma \downarrow_{l} \\
e \downarrow_{l} & =\text { nop } \\
p s^{\prime} \downarrow_{l} & =\{\}
\end{aligned}
$$

Proof. Omitted.

\section{Lemma (Projection 1).}

(1) If $\Sigma \stackrel{e_{1}}{\longrightarrow} \Sigma_{1}^{\prime}$, Proof.

then $\exists \Sigma_{2}^{\prime} \cdot \exists e_{2} . \Sigma \downarrow_{l} \stackrel{e_{2}}{\longrightarrow} \Sigma_{2}^{\prime}$ and $\Sigma_{1}^{\prime} \approx_{l} \Sigma_{2}^{\prime}$ and $e_{1}^{\prime} \approx_{l} e_{2}^{\prime}$.

Let $(\sigma, p s)=\Sigma$.

Let $\left(\sigma_{1}^{\prime}, p s_{1}^{\prime}\right)=\Sigma_{1}^{\prime}$.

Proceed by cases (ie inversion) on (1).

Case: [s-sTART]. Let $t, l^{\prime}$ be such that:

$e_{1}=\operatorname{start}\left(t, l^{\prime}\right) ;$ and

$\sigma_{1}^{\prime}=\sigma$; and

$p s_{1}^{\prime}=p s+\left(t, l^{\prime}\right)$.

Proceed by cases.

Case: where $l^{\prime} \sqsubseteq l$.

Pick $\Sigma_{2}^{\prime}=\left(\sigma \downarrow_{l}, p s \downarrow_{l}+\left(t, l^{\prime}\right)\right)$.

Pick $e_{2}^{\prime}=\operatorname{start}\left(t, l^{\prime}\right)$.

QED

by [S-START]. 
Case: where $l^{\prime} \nsubseteq l$.

Pick $\Sigma_{2}^{\prime}=\Sigma \downarrow_{l}$ and pick $e_{2}^{\prime}=\epsilon$.

QED

by [S-SKIP].

Case: [s-skip]. $e_{1}=\epsilon$ and $\Sigma_{1}^{\prime}=\Sigma$.

Pick $\Sigma_{2}^{\prime}=\Sigma \downarrow_{l}$ and pick $e_{2}^{\prime}=\epsilon$.

QED

by [s-SKIP].

Last case: any other rule. Let $t, l^{\prime}, p s_{1}, p s_{2}$ be such that:

$p s=p s_{1} \uplus\left\{\left(t, l^{\prime}\right)\right\}$; and

$p s_{1}^{\prime}=p s_{1} \uplus p s_{2}$; and

Proceed by cases.

Case: where $l^{\prime} \nsubseteq l$.

$\sigma_{1}^{\prime} \downarrow_{l}=\sigma_{l}$ and $e_{1} \downarrow_{l}=\epsilon$ and $p s_{2} \downarrow_{l}=\{\} \quad$ by Invisibility.

QED by [s-SKIP].

Last case: where $l^{\prime} \sqsubseteq l$.

Resume case analysis on (1).

Case: [s-SEND]. Let $c h, v, t^{\prime}$ be such that:

$e_{1}=$ output $\operatorname{ch} v$; and

$\sigma_{1}^{\prime}=\sigma ;$ and

$p s_{2}=\left\{\left(t^{\prime}, l^{\prime}\right)\right\} ;$ and

$\operatorname{run}(t)=\operatorname{send} \operatorname{ch} v t^{\prime}$; and

$l^{\prime} \sqsubseteq \operatorname{label}(\mathrm{ch})$.

Pick $\Sigma_{2}^{\prime}=\left(\sigma \downarrow_{l}, p s_{1} \downarrow_{l} \uplus\left\{\left(t^{\prime}, l^{\prime}\right)\right\}\right)$.

Pick $e_{2}^{\prime}=$ output $\operatorname{ch} v$.

QED

by [S-STEP].

Remaining cases omitted.

\section{Lemma (Projection 2).}

(1) If $\Sigma \downarrow_{l} \stackrel{e_{1}}{\longrightarrow} \Sigma_{1}$,

then $\exists \Sigma_{2} . \exists e_{2} . \Sigma \stackrel{e_{2}}{\longrightarrow} \Sigma_{2}$ and $\Sigma_{1} \approx_{l} \Sigma_{2}$ and $e_{1} \approx_{l} e_{2}$.

Proof.

Let $(\sigma, p s)=\Sigma$.

Let $\left(\sigma_{1}, p s_{1}\right)=\Sigma_{1}$.

Proceed by cases (ie inversion) on (1).

Case: [s-START]. Let $t, l^{\prime}$ be such that:

$e_{1}=\left(t, l^{\prime}\right)$; and

$\sigma_{1}=\sigma \downarrow_{l} ;$ and

$p s_{1}=p s \downarrow_{l}+\left(t, l^{\prime}\right)$; and

Pick $\Sigma_{2}=\left(\sigma, p s+\left(t, l^{\prime}\right)\right)$ and $e_{2}=e_{1}$.

QED

by [S-START].

Case: [s-SKIP].

Pick $\Sigma_{2}=\Sigma$ and $e_{2}=\epsilon$.

QED by [s-SKIP].

Case: [s-SEND]. Let $t, l^{\prime}, p s_{3}, c h, v, t^{\prime}$ be such that:

(2) $\quad p s \downarrow_{l}=p s_{3} \uplus\left\{\left(t, l^{\prime}\right)\right\}$; and

$p s_{1}=p s_{3} \uplus\left\{\left(t^{\prime}, l^{\prime}\right)\right\}$; and

$e_{1}=$ output $c h v$; and 


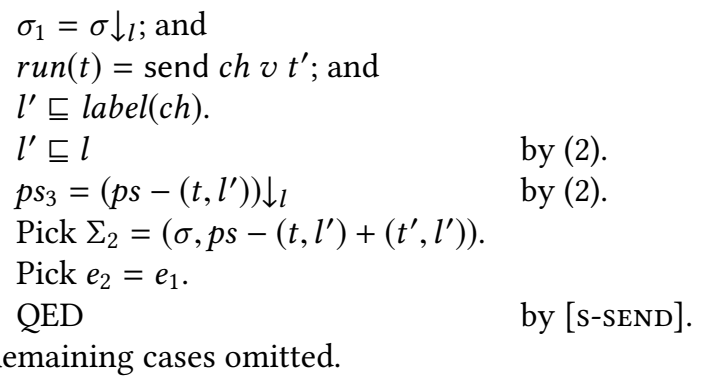

\section{ACKNOWLEDGMENTS}

We thank Aurojit Panda, David Tennenhouse, and the anonymous referees for insightful comments that improved this paper. This publication is part of projects that have received funding from the European Research Council (ERC) under the European Union's Seventh Framework Program (FP7/2007-2013) / ERC grant agreement no. [321174-VSSC]. The research was supported in part by Len Blavatnik and the Blavatnik Family foundation, the Blavatnik Interdisciplinary Cyber Research Center at the Tel Aviv University, and the Pazy Foundation. This material is based upon work supported by the United States-Israel Binational Science Foundation (BSF) grants No. 2016260. This research was supported, in part, by NSF Grants 1337278 and 1421016. SF and KW were supported by NSF grant CNS-1528197, DARPA grant HR0011-15-2-0047, and by VMware, Google, Huawei, Dropbox, and Facebook.

\section{REFERENCES}

Airbnb. 2017. StreamAlert: A serverless framework for real-time data analysis and alerting. http://airbnb.io/projects/ streamalert/.

Kalev Alpernas, Cormac Flanagan, Sadjad Fouladi, Leonid Ryzhyk, Mooly Sagiv, Thomas Schmitz, and Keith Winstein. 2017. Trapeze source code repository. https://github.com/kalevalp/trapeze.

Amazon. 2017a. AWS Lambda. https://aws.amazon.com/lambda/.

Amazon. 2017b. AWS Rekognition. https://aws.amazon.com/rekognition/.

Apache Software Foundation. [n. d.]. Apache Hadoop. https://hadoop.apache.org/.

Apache Software Foundation. 2017. OpenWhisk. https://openwhisk.apache.org/.

Aslan Askarov, Sebastian Hunt, Andrei Sabelfeld, and David Sands. 2008. Termination-Insensitive Noninterference Leaks More Than Just a Bit. In Proc. of ESORICS 2008. Malaga, Spain, 333-348.

Thomas H. Austin and Cormac Flanagan. 2009. Efficient Purely-dynamic Information Flow Analysis. In Proc. of PLAS 2009. 113-124.

Thomas H. Austin and Cormac Flanagan. 2010. Permissive Dynamic Information Flow Analysis. In Proc. of PLAS 2010. 1-12.

Thomas H. Austin and Cormac Flanagan. 2012. Multiple Facets for Dynamic Information Flow. In Proc. of POPL 2012. 165-178.

Thomas H. Austin, Tommy Schmitz, and Cormac Flanagan. 2017. Multiple Facets for Dynamic Information Flow with Exceptions. ACM Trans. Program. Lang. Syst. 39, 3, Article 10 (May 2017), 56 pages. https://doi.org/10.1145/3024086

Thomas H. Austin, Jean Yang, Cormac Flanagan, and Armando Solar-Lezama. 2013. Faceted Execution of Policy-agnostic Programs. In Proc. of PLAS. Seattle, Washington, USA, 15-26.

Jean Bacon, David Eyers, Thomas F. J.-M. Pasquier, Jatinder Singh, Ioannis Papagiannis, and Peter Pietzuch. 2014. Information Flow Control for Secure Cloud Computing. IEEE Transactions on Network and Service Management 11, 1 (Jan. 2014), 76-89.

Andrew Baird, Michael Connor, and Patrick Brandt. 2016. Coca-Cola: Running Serverless Applications with Enterprise Requirements. https://aws.amazon.com/serverless/videos/video-lambda-coca-cola/.

D. Elliott Bell and Leonard J. LaPadula. 1973. Secure Computer Systems: Mathematical Foundations. Technical Report 2547. MITRE.

Nataliia Bielova and Tamara Rezk. 2016. Spot the difference: Secure multi-execution and multiple facets. In European Symposium on Research in Computer Security. Springer, 501-519.

Arnab Kumar Biswas, Dipak Ghosal, and Shishir Nagaraja. 2017. A Survey of Timing Channels and Countermeasures. ACM Comput. Surv. 50, 1 (March 2017), 6:1-6:39. 
Aaron Bohannon, Benjamin C Pierce, Vilhelm Sjöberg, Stephanie Weirich, and Steve Zdancewic. 2009. Reactive noninterference. In Proceedings of the 16th ACM conference on Computer and communications security. ACM, 79-90.

Mark Boyd. 2017. iRobot Confronts the Challenges of Running Serverless at Scale. https:/thenewstack.io/ irobot-confronts-challenges-running-serverless-scale/.

Fraser Brown, Shravan Narayan, Riad S. Wahby, Dawson Engler, Ranjit Jhala, and Deian Stefan. 2017. Finding and Preventing Bugs in JavaScript Bindings. In Proc. of S\&P 2017. 559-578.

Kuldeep Chowhan. 2016. Serverless Computing Patterns at Expedia. https:/www.slideshare.net/AmazonWebServices/ aws-reinvent-2016-serverless-computing-patterns-at-expedia-svr306.

CNET Magazine. 2011. The PlayStation Network breach (FAQ). https://www.cnet.com/news/ the-playstation-network-breach-faq/.

Computerworld. 2009. SQL injection attacks led to Heartland, Hannaford breaches. https://www.computerworld.com/ article/2527185/security0/sql-injection-attacks-led-to-heartland--hannaford-breaches.html.

Computerworld. 2014. Two-factor authentication oversight led to JPMorgan breach. https://www.computerworld.com/ article/2862578/twofactor-authentication-oversight-led-to-jpmorgan-breach-investigators-reportedly-found.html.

Willem De Groef, Dominique Devriese, Nick Nikiforakis, and Frank Piessens. 2012. FlowFox: a web browser with flexible and precise information flow control. In Proc. of CCS 2012. 748-759.

Dorothy E Denning. 1976. A lattice model of secure information flow. Comm, of the ACM 19, 5 (1976), 236-243.

Dorothy E. Denning and Peter J. Denning. 1977. Certification of Programs for Secure Information Flow. Commun. ACM 20, 7 (July 1977), 504-513.

Dominique Devriese and Frank Piessens. 2010. Noninterference Through Secure Multi-execution. In Proc. IEEE SSP 2010. 109-124.

Digital Trends. 2016. The latest data breach involves the voting records of 93.4 million Mexican citizens. https://www. digitaltrends.com/computing/mexico-voting-breach/.

Petros Efstathopoulos, Maxwell Krohn, Steve VanDeBogart, Cliff Frey, David Ziegler, Eddie Kohler, David Mazières, Frans Kaashoek, and Robert Morris. 2005. Labels and event processes in the Asbestos operating system. In Proc. of SOSP 2005.

Ken Ellis. 2017. How Reuters Replaced WebSockets with Amazon Cognito and SQS. https://serverless.com/blog/ how-reuters-replaced-websockets-with-amazon-cognito-and-sqs/.

Marius Eriksen. 2013. Your server as a function. In In Proc. of PLOS 2013.

Fn Project. 2017. https://fnproject.io/.

Forbes. 2014. eBay Suffers Massive Security Breach, All Users Must Change Their Passwords. https://www.forbes. com/sites/gordonkelly/2014/05/21/ebay-suffers-massive-security-breach-all-users-must-their-change-passwords/ \#793467c57492.

Forbes. 2017. How Hackers Broke Equifax: Exploiting A Patchable Vulnerability. https://www.forbes.com/sites/ thomasbrewster/2017/09/14/equifax-hack-the-result-of-patched-vulnerability/\#20abe9015cda.

Sadjad Fouladi, Dan Iter, Shuvo Chatterjee, Christos Kozyrakis, Matei Zaharia, and Keith Winstein. 2017a. A Thunk to Remember: make -j1000 (and other jobs) on functions-as-a-service infrastructure (Under review). http://stanford.edu/ $\sim$ sadjad/gg-paper.pdf.

Sadjad Fouladi, Riad S. Wahby, Brennan Shacklett, Karthikeyan Vasuki Balasubramaniam, William Zeng, Rahul Bhalerao, Anirudh Sivaraman, George Porter, and Keith Winstein. 2017b. Encoding, Fast and Slow: Low-Latency Video Processing Using Thousands of Tiny Threads. In Proc. of NSDI 2017. Boston, MA, 363-376.

gg project. 2017. gg source code repository. https://github.com/stanfordsnr/gg.

Google. 2017. Google Cloud Functions. https://cloud.google.com/functions/.

Nevin Heintze and Jon G. Riecke. 1998. The SLam Calculus: Programming with Secrecy and Integrity. In Proc. of POPL 1998. San Diego, California, USA, 365-377.

Tyler Hunt, Zhiting Zhu, Yuanzhong Xu, Simon Peter, and Emmett Witchel. 2016. Ryoan: A Distributed Sandbox for Untrusted Computation on Secret Data. In Proc. of OSDI 2016. Savannah, GA, USA, 533-549.

IBM. 2017. IBM Cloud Functions. https://console.bluemix.net/openwhisk/.

Intel Corporation. 2014. Intel Software Guard Extensions Programming Reference.

Eric Jonas, Shivaram Venkataraman, Ion Stoica, and Benjamin Recht. 2017. Occupy the Cloud: Distributed Computing for the 99\%. CoRR abs/1702.04024 (2017). http://arxiv.org/abs/1702.04024

Murad Kablan, Azzam Alsudais, Eric Keller, and Franck Le. 2017. Stateless Network Functions: Breaking the Tight Coupling of State and Processing. In Proc. of NSDI 2017. Boston, MA, 97-112.

Vineeth Kashyap, Ben Wiedermann, and Ben Hardekopf. 2011. Timing-and termination-sensitive secure information flow: Exploring a new approach. In 2011 IEEE Symposium on Security and Privacy. IEEE, 413-428.

McKim, John. 2017. Announcing the Winners of the Inaugural ServerlessConf Architecture Competition. https://read. acloud.guru/announcing-the-winners- of-the-inaugural-serverlessconf-architecture-competition-1dce2db6da3.

Microsoft. 2017. Azure Functions. https://azure.microsoft.com/services/functions/. 
Andrew C Myers. 1999. JFlow: Practical mostly-static information flow control. In Proc. of POPL 1999. 228-241.

Andrew C Myers and Barbara Liskov. 2000. Protecting privacy using the decentralized label model. TOSEM 9, 4 (2000), 410-442.

National Vulnerability Database. 2017. CVE-2017-5638. https:/nvd.nist.gov/vuln/detail/CVE-2017-5638.

nodemailer 2017. nodemailer. https://github.com/nodemailer/nodemailer.

Nordstrom Technology. 2017. Hello, Retail! https:/github.com/Nordstrom/hello-retail.

Thomas Pasquier, Jean Bacon, Jatinder Singh, and David Eyers. 2016. Data-Centric Access Control for Cloud Computing. In Proc. of SACMAT 2016. Shanghai, China, 81-88.

PCWorld. 2010. Microsoft Cloud Data Breach Heralds Things to Come. https://www.pcworld.com/article/214775/microsoft cloud_data_breach_sign_of_future.html.

Andrei Sabelfeld and Andrew C Myers. 2003. Language-based information-flow security. IEEE fournal on selected areas in communications 21, 1 (2003), 5-19.

Andrei Sabelfeld and David Sands. 2001. A Per Model of Secure Information Flow in Sequential Programs. Higher Order Symbol. Comput. 14, 1 (March 2001), 59-91.

Peter Sbarski. 2017. Serverless Architectures on AWS: With examples using AWS Lambda. Manning Publications, Shelter Island, NY.

Serverless, Inc. 2017. Serverless Examples. https://github.com/serverless/examples.

Geoffrey Smith and Dennis Volpano. 1998. Secure Information Flow in a Multi-threaded Imperative Language. In Proc. of POPL 1998. San Diego, California, USA, 355-364.

Deian Stefan, Alejandro Russo, Pablo Buiras, Amit Levy, John C Mitchell, and David Mazieres. 2012. Addressing covert termination and timing channels in concurrent information flow systems. In ACM SIGPLAN Notices, Vol. 47. 201-214.

Deian Stefan, Alejandro Russo, John C. Mitchell, and David Mazières. 2011. Flexible Dynamic Information Flow Control in Haskell. In Proc. of Haskell 2011. 95-106.

TechRepublic. 2017. Massive Amazon S3 leaks highlight user blind spots in enterprise race to the cloud. https://www. techrepublic.com/article/massive-amazon-s3-breaches-highlight-blind-spots-in-enterprise-race-to-the-cloud/.

The Register. 2011. RSA explains how attackers breached its systems. https:/www.theregister.co.uk/2011/04/04/rsa_hack_ howdunnit/.

Tom Van Cutsem and Mark S. Miller. 2013. Trustworthy Proxies: Virtualizing Objects with Invariants. In Proc. of ECOOP 2013. Montpellier, France, 154-178.

VM2 2017. VM2. https://github.com/patriksimek/vm2.

Wikipedia. 2017a. Anthem medical data breach. https://en.wikipedia.org/wiki/Anthem_medical_data_breach.

Wikipedia. 2017b. Sony Pictures hack. https://en.wikipedia.org/wiki/Sony_Pictures_hack.

Wikipedia. 2017c. Yahoo! data breaches. https://en.wikipedia.org/wiki/Yahoo!_data_breaches.

Wired. 2016. Inside the Cyberattack That Shocked the US Government. https://www.wired.com/2016/10/ inside-cyberattack-shocked-us-government/.

Jean Yang, Travis Hance, Thomas H. Austin, Armando Solar-Lezama, Cormac Flanagan, and Stephen Chong. 2016. Precise, Dynamic Information Flow for Database-backed Applications. In Proc. of PLDI 2016. Santa Barbara, CA, USA.

Matei Zaharia, Mosharaf Chowdhury, Tathagata Das, Ankur Dave, Justin Ma, Murphy McCauley, Michael J. Franklin, Scott Shenker, and Ion Stoica. 2012. Resilient Distributed Datasets: A Fault-tolerant Abstraction for In-memory Cluster Computing. In Proc. of NSDI 2012. San Jose, CA.

Stephan Arthur Zdancewic. 2002. Programming languages for information security. Ph.D. thesis, Cornell University.

ZDNet. 2015. Anatomy of the Target data breach: Missed opportunities and lessons learned. http://www.zdnet.com/article/ anatomy-of-the-target-data-breach-missed-opportunities-and-lessons-learned/.

ZDNet. 2016. AdultFriendFinder network hack exposes 412 million accounts. http://www.zdnet.com/article/ adultfriendfinder-network-hack-exposes-secrets-of-412-million-users. 